\title{
Selection of a Forwarding Area for Contention-Based Geographic Forwarding in Wireless Multi-Hop Networks
}

\author{
Dazhi Chen, Member, IEEE, Jing Deng, Member, IEEE, and Pramod K. Varshney, Fellow, IEEE
}

\begin{abstract}
Contention-based Geographic Forwarding (CGF) is a state-free forwarding technique. In this paper, we develop a general analytical framework to evaluate the performance of CGF with different forwarding areas in wireless multi-hop networks. In particular, we compare the performance of CGF for three typical forwarding areas, analytically and by extensive simulations. We further investigate the impact of several important assumptions on our analytical results. Our study provides guidelines regarding the selection of a specific forwarding area during the design phase of a CGF protocol. It also serves as a general performance evaluation framework for CGF protocols as well as traditional geographic forwarding protocols.
\end{abstract}

Index Terms-Ad hoc and sensor networks, geographic forwarding, performance evaluation, void handling, wireless networks.

\section{INTRODUCTION}

G EOGRAPHIC routing, consisting of a geographic forwarding strategy and a location service, has been established as a promising solution for information delivery in next-generation wireless networks [1]. In traditional geographic forwarding, a node acquires the geographic information of neighbors by a periodic beaconing scheme. The scheme incurs unnecessary overhead when there is no data traffic or when the neighborhood information is still fresh. It also results in performance deterioration when the network is highly dynamic.

To eliminate the proactive beaconing scheme, some researchers [2]-[7] have solved this problem recently using the key idea of instant access to the information regarding the neighbors and distributed selection of a next-hop node via contention. In this paper, we term such techniques as Contentionbased Geographic Forwarding (CGF).

While the existing CGF protocols differ in protocol details, the basic procedure remains the same: Whenever a sender needs to forward a data packet, it broadcasts a control packet requesting to send its data packet to its direct neighbors. Upon

Manuscript received November 22, 2005; revised October 18, 2006 and December 5, 2006. The review of this paper was coordinated by Dr. W. Zhuang.

D. Chen and P. K. Varshney are with the Department of Electrical Engineering and Computer Science, Syracuse University, Syracuse, NY 13244 USA (e-mail: dchen02@syr.edu; varshney@syr.edu).

J. Deng is with the Department of Computer Science, University of New Orleans, New Orleans, LA 70148 USA (e-mail: jing@cs.uno.edu).

Color versions of one or more of the figures in this paper are available online at http://ieeexplore.ieee.org.

Digital Object Identifier 10.1109/TVT.2007.900371 receiving the request, every neighbor independently determines whether it can forward the data packet according to its position and other information, and if so, it contends for the task based on some rules. The neighbors that are eligible to forward the data packet, e.g., they are within a forwarding area that is expected to make a positive geographic progress toward the destination, are called next-hop candidates. When a neighbor wins the contention, it confirms this fact with the sender, and other neighbors quit the current contention. The sender then unicasts the actual data packet to the winner. If such a nexthop node in the forwarding area does not exist, which is known as a communication void or local minimum phenomenon [8], a void handling scheme is employed to route the packet around the void. This process is repeated until the packet is successfully delivered to its destination.

Accordingly, CGF mainly consists of the following components: 1) A predefined forwarding area and nodes that reside in the area become next-hop candidate nodes; 2) a distributed contention arbitration and resolution scheme to effectively establish a single next-hop node in the forwarding area; 3) a next-hop node selection criterion so as to attain the desired network performance efficiently; and 4) an effective mechanism to handle voids.

In the existing CGF protocols, forwarding areas of different sizes, shapes, and geographic locations have been employed, leading to several intriguing research issues: Given a specified forwarding area, how can we analyze the network performance of CGF? Which performance metrics should be used? How do different forwarding areas affect the performance of CGF? Which of the forwarding areas currently in use performs the best? How to select a forwarding area a priori when designing a new CGF protocol? Intuitively, the forwarding area influences the progress a packet makes toward the destination during a single hop. Furthermore, it also affects the design of the contention resolution mechanism and the possibility of voids that may lead to undesired packet loss in the network.

Our work attempts to answer the aforementioned research issues, and thus, in this paper, we focus on the study of a predefined forwarding area for CGF via theoretical analysis and through extensive simulations. To the best of our knowledge, our work is the first study on CGF from this perspective. The contributions of this paper are given here.

- A high-level model of CGF is established. This model can be used to analyze the network performance of a broad range of geographic routing protocols. 


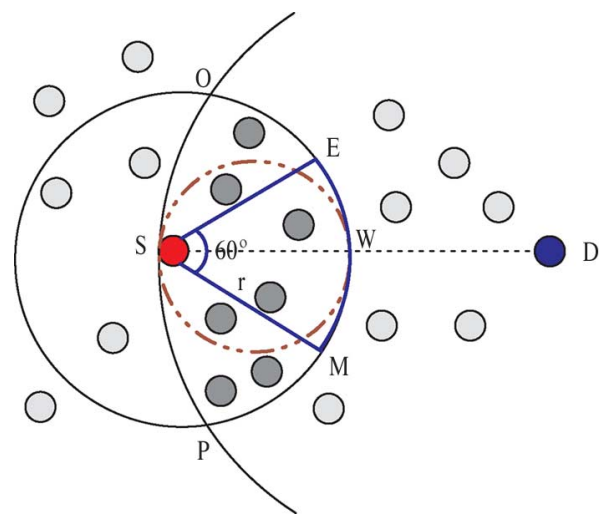

Fig. 1. Typical forwarding areas for CGF.

- A general mathematical analysis framework, in terms of average single-hop progress and average successful delivery probability, is developed to evaluate CGF with different forwarding areas of interest.

- The detailed performance comparison of three typical forwarding areas in the existing CGF protocols. Our results provide insights into the projected performance of the CGF protocols using these areas.

- A simulation-based investigation of the impact of several important assumptions on our numerically derived results.

The rest of this paper is organized as follows. In Section II, we define and discuss three typical forwarding areas. A general analytical framework for the performance of CGF with different forwarding areas is presented in Section III. Section IV provides numerical and simulation results along with our discussion. Section V further discusses several important assumptions. We conclude our work in Section VI.

\section{FORWARDING AREAS IN CGF}

All the forwarding areas in CGF have a common requirement in that each transmission should make a positive progress toward the destination (unless a void occurs and such a transmission cannot take place). By assuming that the transmission range of a node is in the form of a perfect circle, we define three typical forwarding areas, as shown in Fig. 1, for CGF.

1) Maximum Forwarding Area (MFA). It is the overlap region of two circular areas: 1) the transmission circle of the sender and 2) the circle that is centered at the destination with a radius equal to the distance between the sender and the destination. It is the region OSPW that is shown in Fig. 1. The size of this area depends on the transmission radius and the distance between the sender and the destination. MFA has been adopted in the GeRaF [4], the SIF [6], and the MACRO [7] protocols.

2) Maximum Communication Area (MCA). It is defined as the largest region within which any pair of nodes can hear each other [2], [3]. Thus, it is a circle with a diameter equal to the transmission range of a node. It is the circle with SW as its diameter in Fig. 1. Note that SW should be colinear with SD. Such an arrangement of SW maximizes the possible area within the circle.

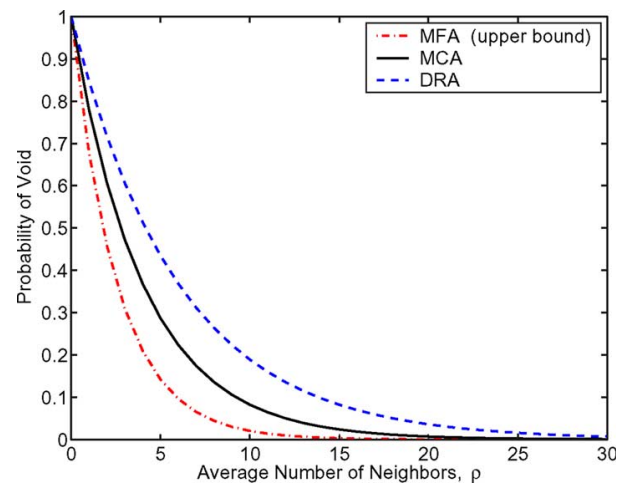

Fig. 2. Probability of void for the three forwarding areas.

3) $60^{\circ}$ Radian Area (DRA). It is a radial region that includes a $30^{\circ}$ radian area around the line connecting the sender and the destination on both sides. It is the region SEM that is shown in Fig. 1. Note that SD is the angle bisector for this region. DRA was used in the IGF [5] and BLR [2] protocols.

The probability of void for each forwarding area is studied next. The node distribution in the region of interest can be modeled as a 2-D Poisson point process. Thus, the probability that $k$ nodes are located within an area of size $A$ is given by [13]

$$
\operatorname{Pr}\{k\}=\frac{(\lambda A)^{k} \cdot e^{-\lambda A}}{k !}
$$

where $\lambda$ is the expected number of nodes within a unit area.

Since the forwarding areas of MCA and DRA are $\pi r^{2} / 4$ and $\pi r^{2} / 6$, respectively, the probabilities of void $(\operatorname{Pr}\{k=0\})$ for MCA and DRA are as follows:

$$
\begin{aligned}
& \operatorname{Pr}\{\text { void in MCA }\}=e^{-\frac{\rho}{4}} \\
& \operatorname{Pr}\{\text { void in DRA }\}=e^{-\frac{\rho}{6}}
\end{aligned}
$$

where $\rho$ is the average number of neighbors within the transmission range $r$ of the sender and is given by

$$
\rho=\pi r^{2} \lambda \text {. }
$$

The probability of void for the MFA area can be upper bounded by (cf. [6])

$$
\operatorname{Pr}\{\text { void in MFA }\} \leq e^{-\left(\frac{2}{3}-\frac{\sqrt{3}}{2 \pi}\right) \rho} \approx e^{-\frac{\rho}{2.5}} .
$$

The results from (2), (3), and (5) are plotted in Fig. 2. From the figure, it can be observed that the upper bound on the probability of void of MFA is lower than the probability of void of MCA and DRA. For instance, when there are, on an average, ten nodes within each node's transmission range $(\rho=10)$, the upper bound on the probability of void in MFA is 0.02 , whereas the probabilities of void for MCA and DRA are 0.082 and 0.189 , respectively. Note that when $\rho$ is higher than 30 , the probabilities of void for all the three forwarding areas are close to 0 . This suggests that at least one next-hop node will be found in each of the three forwarding areas.

Although MFA has a lower probability of void than MCA and DRA, a more interesting and challenging issue is: Does 
CGF employing MFA perform better than CGF that uses MCA or DRA in a randomly deployed network? If so, by how much? The latter question raises the interesting performance metric issue of how to quantify the CGF performance with different forwarding areas. In Section III, we define two performance metrics and model CGF as a general three-step forwarding strategy. A general analytical framework is then developed, and closed-form expressions for the performance metrics of the aforementioned three forwarding areas are derived.

\section{Theoretical ANALYsis}

\section{A. Performance Metrics}

Two metrics are proposed to evaluate the performance of CGF.

1) Average Single-Hop Progress: It is defined as the expected value of the difference between the before-hop distance (between the sender node and the destination node) and the after-hop distance (between the next-hop node and the destination node). This metric reflects the progress toward the intended destination at each hop and has a direct connection with the average packet delay performance of CGF in the long term.

2) Average Successful Delivery Probability: It is defined as the expected value of the frequency of the event that a packet is successfully delivered to the destination node from the source node. This metric reflects the robustness of packet delivery of CGF over multiple hops in the presence of void. ${ }^{1}$

Together, these metrics characterize the performance of CGF.

\section{B. Assumptions and Three-Step Forwarding Strategy}

We make the following assumptions for our study in this paper: A homogeneous wireless multi-hop network is deployed with randomly distributed nodes, where all nodes are stationary and have the common transmission range in the form of a perfect circle of radius $r$. The node distribution can be modeled as in (1), and the appearance of nodes in any two nonoverlapping areas is independent. Nodes adopt the CGF protocol to communicate with each other, and the forwarding area in CGF is specified a priori. A source node $S$ is located at the center of a circle of radius $x$, where $x$ is termed the network range and is the largest possible distance between $S$ and any destination node. A destination node $D$, to which $S$ intends to deliver a data packet, is assumed to be uniformly distributed over the entire circle. If the destination node is located outside the transmission range of the source node, a data packet has to be forwarded over a certain number of intermediate hops before reaching its destination.

We also assume the availability of a collision-free media access scheme and that each node can determine its own location and the location of packet destination without errors. We further

\footnotetext{
${ }^{1}$ Note that we focus on packet loss due to the void problem only; some other factors, e.g., packet collisions at the MAC layer and packet loss due to network congestion, are not considered in this metric.
}

assume that CGF has a contention scheme which perfectly establishes a single next-hop node in a predefined forwarding area, that CGF employs a distance-based greedy criterion which allows the candidate node closest to the destination in the forwarding area to become the next-hop node to forward the packet, and that no void handling scheme is employed (i.e., in the presence of voids). The impact of these assumptions will be further discussed in Section V.

Based on the aforementioned assumptions, CGF can be modeled as a three-step forwarding strategy. ${ }^{2}$

1) If the destination node $D$ is located within distance $r$ from the source node $S, D$ always wins the contention, and $S$ delivers the data packet to $D$ directly.

2) If $D$ is outside the transmission range of $S$, the data packet is always forwarded by a next-hop node that is closest to $D$ in the forwarding area.

3) If there are no next-hop candidate nodes in the forwarding area, $S$ does not forward the data packet and discards it after a predefined time period during which no candidate nodes appear in the forwarding area.

\section{Average Single-Hop Progress}

Consider the snapshot at the time of the first-hop packet forwarding, even if multi-hop forwarding is required for data packets to arrive at their destinations. Given a random node deployment, any intermediate-hop data forwarding can be viewed as a new first-hop packet forwarding. Thus, the first-hop average progress is a good approximation to the overall average single-hop progress in a homogeneous environment.

Let $v$ denote the distance between the source node $S$ and the destination node $D$. When $v \leq r$, direct data delivery to the destination node $D$ can be completed, according to step 1 in the forwarding strategy; hence, the data packet progress toward the destination node $D$ is $v$. If $v>r$, an appropriate (i.e., nearest to the destination in this context, according to step 2 in the forwarding strategy) next-hop node in its forwarding area is selected for further packet forwarding. The data packet progress toward the destination node $D$ is thereby equal to $(v-n)$, where $n$ is the distance between the next-hop node $F$ and the destination node $D$ corresponding to the first hop.

Denote by $\boldsymbol{P}$ the random variable corresponding to the packet progress in a single hop. Let $\boldsymbol{V}$ and $\boldsymbol{N}$ be the random variables corresponding to $v$ and $n$, respectively. $\boldsymbol{I}$ is an index random variable, which is defined as

$$
\boldsymbol{I}= \begin{cases}1, & \text { at least one node in the forwarding area } \\ 0, & \text { otherwise }\end{cases}
$$

With the aforementioned notations, we can convert the descriptive three-step forwarding strategy into the following

\footnotetext{
${ }^{2}$ Note that, if MFA is employed in CGF, the three-step forwarding strategy with a slightly different description can also be used to model traditional geographic forwarding [11]. As a result, our analytical framework presented in this paper can be applied to evaluate the performance of traditional geographic forwarding as well.
} 


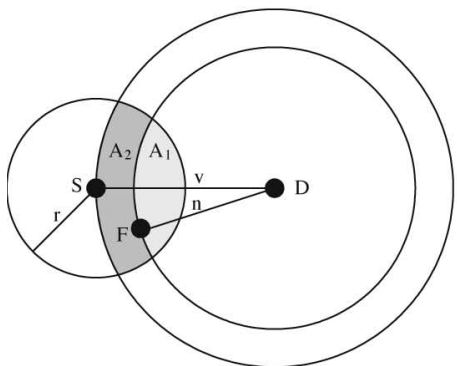

(a)

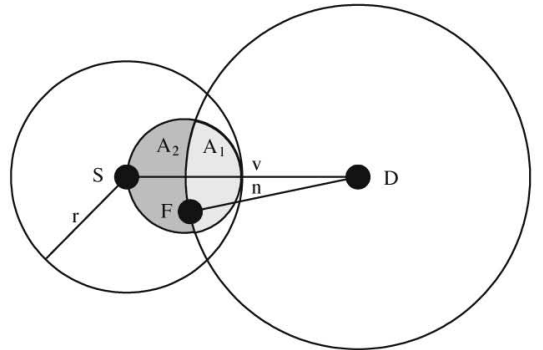

(b)

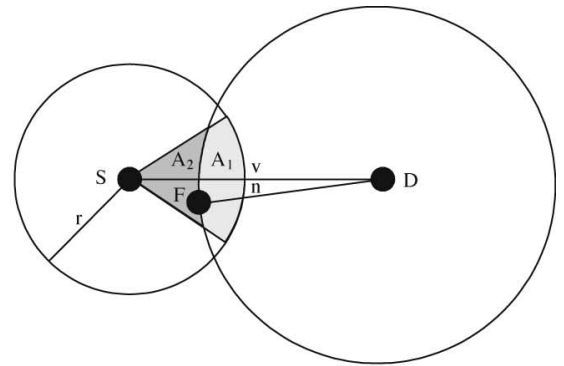

(c)

Fig. 3. Illustration of forwarding areas. (a) MFA. (b) MCA. (c) DRA.

mathematical expression:

$$
\boldsymbol{P}= \begin{cases}\boldsymbol{V}, & \text { if }(\boldsymbol{V} \leq r) \\ \boldsymbol{V}-\boldsymbol{N}, & \text { if }(\boldsymbol{V}>r) \cap(\boldsymbol{I}=1) \\ 0, & \text { if }(\boldsymbol{V}>r) \cap(\boldsymbol{I}=0)\end{cases}
$$

Note that, if $(\boldsymbol{V}>r \cap \boldsymbol{I}=0)$ is true, no packet forwarding will occur. In this case, the actual progress is 0 . It can, therefore, be verified that

$$
\begin{aligned}
E[\boldsymbol{P}]=\operatorname{Pr}\{(\boldsymbol{V} & \leq r) \cup(\boldsymbol{V}>r \cap \boldsymbol{I}=1)\} \\
& \times E[\boldsymbol{P} \mid(\boldsymbol{V} \leq r) \cup(\boldsymbol{V}>r \cap \boldsymbol{I}=1)]
\end{aligned}
$$

We now proceed to derive $E[\boldsymbol{P} \mid(\boldsymbol{V} \leq r) \cup(\boldsymbol{V}>r \cap$ $\boldsymbol{I}=1)$ ]. From [11], we have

$$
\begin{aligned}
\operatorname{Pr} & \{\boldsymbol{P}>p \mid(\boldsymbol{V} \leq r) \cup(\boldsymbol{V}>r \cap \boldsymbol{I}=1)\} \\
= & \frac{\operatorname{Pr}\{p<\boldsymbol{V} \leq r\}+\operatorname{Pr}\{\boldsymbol{V}-\boldsymbol{N}>p \cap \boldsymbol{V}>r \cap \boldsymbol{I}=1\}}{\operatorname{Pr}\{\boldsymbol{V} \leq r\}+\operatorname{Pr}\{\boldsymbol{V}>r \cap \boldsymbol{I}=1\}} .
\end{aligned}
$$

We therefore need to determine four probability expressions in (8). Among them, $\operatorname{Pr}\{p<\boldsymbol{V} \leq r\}$ and $\operatorname{Pr}\{\boldsymbol{V} \leq r\}$ can be easily determined as follows.

$$
\begin{gathered}
\operatorname{Pr}\{p<\boldsymbol{V} \leq r\}=\frac{\left(r^{2}-p^{2}\right)}{x^{2}} \boldsymbol{U}(r-p) \\
\operatorname{Pr}\{\boldsymbol{V} \leq r\}=\frac{r^{2}}{x^{2}}
\end{gathered}
$$

where $\boldsymbol{U}(\cdot)$ is defined as

$$
\boldsymbol{U}(r-p)= \begin{cases}1, & \text { if } r \geq p \\ 0, & \text { if } r<p\end{cases}
$$

Let $A_{S D}$ denote a general forwarding area. Furthermore, we can divide $A_{S D}$ into two regions by the circle centered at $D$ with radius $n$. The areas of these two regions are denoted as $A_{1}$ and $A_{2}$, respectively, as the shaded regions that are shown in Fig. 3. As we have derived in [10], $\operatorname{Pr}\{\boldsymbol{V}>r \cap \boldsymbol{I}=1\}$ and $\operatorname{Pr}\{\boldsymbol{V}-\boldsymbol{N}>p \cap \boldsymbol{V}>r \cap \boldsymbol{I}=1\}$ are determined as follows:

$$
\operatorname{Pr}\{\boldsymbol{V}>r \cap \boldsymbol{I}=1\}=1-\frac{r^{2}}{x^{2}}-\frac{2}{x^{2}} \int_{r}^{x} v e^{-\lambda A_{S D}} d v
$$

and

$$
\begin{aligned}
\operatorname{Pr}\{\boldsymbol{V}-\boldsymbol{N} & >p \cap \boldsymbol{V}>r \cap \boldsymbol{I}=1\} \\
& =\left(1-\frac{r^{2}}{x^{2}}-\frac{2}{x^{2}} \int_{r}^{x} v e^{-\lambda A_{1}} d v\right) \boldsymbol{U}(r-p) .
\end{aligned}
$$

Finally, substituting (9)-(12) into (8), we have

$$
\begin{aligned}
\operatorname{Pr}\{\boldsymbol{P}>p \mid(\boldsymbol{V} \leq r) \cup(\boldsymbol{V}>r \cap \boldsymbol{I}=1)\} \\
=\left(\frac{x^{2}-p^{2}-2 \int_{r}^{x} v e^{-\lambda A_{1}} d v}{x^{2}-2 \int_{r}^{x} v e^{-\lambda A_{S D} d v}}\right) \boldsymbol{U}(r-p) .
\end{aligned}
$$

The expected value of $\boldsymbol{P}$ given the validity of $(\boldsymbol{V} \leq r) \cup$ $(\boldsymbol{V}>r \cap \boldsymbol{I}=1)$ is then equal to [12, eq. (21.9)]

$$
\begin{aligned}
E[\boldsymbol{P} \mid(\boldsymbol{V} \leq r) \cup(\boldsymbol{V}>r \cap \boldsymbol{I}=1)] \\
=\int_{0}^{\infty} \operatorname{Pr}\{\boldsymbol{P}>p \mid(\boldsymbol{V} \leq r) \cup(\boldsymbol{V}>r \cap \boldsymbol{I}=1)\} d p \\
=\frac{3 x^{2} r-r^{3}-6 \int_{0}^{r} \int_{r}^{x} v e^{-\frac{\rho A_{1}}{\pi r^{2}}} d v d p}{3\left(x^{2}-2 \int_{r}^{x} v e^{-\frac{\rho A_{S D}}{\pi r^{2}}} d v\right)}
\end{aligned}
$$

where $\rho$ is given by (4).

Substituting (10), (11), and (13) into (7), we attain the following general expression for average single-hop progress, independent of any forwarding areas, for networks with CGF:

$$
E[\boldsymbol{P}]=\frac{3 x^{2} r-r^{3}-6 \int_{0}^{r} \int_{r}^{x} v e^{-\frac{\rho A_{1}}{\pi r^{2}}} d v d p}{3 x^{2}} .
$$

Note that the difference between MFA, MCA, and DRA lies in the expressions of $A_{1}$. We have calculated $A_{1}$ for MFA, MCA, and DRA in [10]. Specific average single-hop progress for an individual area is given by (14) after the appropriate $A_{1}$ is substituted.

\section{Asymptotic Behavior of Single-Hop Progress}

When $\rho$ is reasonably large, the expected value of $\boldsymbol{P}$ is

$$
E[\boldsymbol{P}] \rightarrow r-\frac{r^{3}}{3 x^{2}}, \quad \text { for large } \rho,
$$


for any of the three forwarding areas. When $x \gg r$, we have

$$
E[\boldsymbol{P}] \rightarrow r, \quad \text { for large } \rho \text { and } x \gg r .
$$

Thus, average single-hop progress is the same for all forwarding areas. This is expected: As shown in Fig. 1, every forwarding area includes the tip region, which is closest to the destination. As $\rho$ increases to a large value, at least one node exists in this region. The choice of different forwarding areas will not affect the selection of forwarding nodes.

Note that in (15), when $x=r$, the expected progress is equal to $2 r / 3$, as all destinations can be reached from the source within a single hop.

\section{E. Average Successful Delivery Probability}

If the distance $v$ between the source node $S$ and the destination $D$ is less than or equal to $r$, i.e., $v \leq r$, only one hop is required, and a packet will successfully arrive at the destination node with a probability of 1 according to step 1 in the forwarding strategy. Otherwise, if $v>r$, multiple hops are needed according to step 2, and a packet may not be delivered to the destination based on step 3 in the forwarding strategy. Denote by $\boldsymbol{Z}$ the random variable corresponding to the probability of successful delivery. Let $\boldsymbol{T}$ be the random variable corresponding to the probability of successful delivery under the condition that $v>r$. With the aforementioned notations, we have the following expression:

$$
\boldsymbol{Z}= \begin{cases}1, & \text { if } \boldsymbol{V} \leq r \\ \boldsymbol{T}, & \text { if } \boldsymbol{V}>r\end{cases}
$$

It can be verified that

$$
\begin{aligned}
E[\boldsymbol{Z}] & =\operatorname{Pr}\{\boldsymbol{V} \leq r\}(1)+\operatorname{Pr}\{\boldsymbol{V}>r\} E[\boldsymbol{T}] \\
& =\frac{r^{2}}{x^{2}}+\left(1-\frac{r^{2}}{x^{2}}\right) E[\boldsymbol{T}] .
\end{aligned}
$$

We now proceed to derive $E[\boldsymbol{T}]$. Since

$$
E[\boldsymbol{T}]=\int_{r}^{x} E[\boldsymbol{T} \mid \boldsymbol{V}=v] \frac{2 v}{\left(x^{2}-r^{2}\right)} d v
$$

we first need to determine $E[\boldsymbol{T} \mid \boldsymbol{V}=v]$, i.e., $E[\boldsymbol{T}]$ under the condition that the distance $\boldsymbol{V}$ is fixed as a constant and known a priori. Let $\boldsymbol{H}$ denote the number of hops required from the source node to the destination node. The following expression can be established:

$$
E[\boldsymbol{T} \mid \boldsymbol{V}=v]=(1-\operatorname{Pr}\{\text { void }\})^{E[\boldsymbol{H} \mid \boldsymbol{V}=v]} .
$$

Now let us proceed to determine $E[\boldsymbol{H} \mid \boldsymbol{V}=v]$. Let $\boldsymbol{X}_{\boldsymbol{i}}$ denote the random variable corresponding to the per-hop progress at the $i$ th hop on an end-to-end path toward the destination node. Note that the per-hop progress at any of the intermediate hops on an end-to-end path here is different from average single-hop progress that we have derived previously, where the destination node is assumed to be uniformly distributed over the entire circle. The cumulative distribution function of $\boldsymbol{X}_{\boldsymbol{i}}$ can be determined as follows:

$$
F_{\boldsymbol{X}_{\boldsymbol{i}}}(a)= \begin{cases}0, & \text { if } a<0 \\ e^{-\lambda A_{1}(i)}, & \text { if } 0 \leq a \leq r \\ 1, & \text { if } a>r\end{cases}
$$

where $A_{1}(i)$ denotes a region partitioned from a general forwarding area at the $i$ th hop, as shown in Fig. 3, and we account for the fact that the probability of choosing a candidate node in the forwarding area as the next-hop node equals the probability of the $A_{1}(i)$ area being empty. That is, there are no other nodes closer to the destination than the chosen node.

Thus, the expected per-hop progress at the $i$ th hop can be determined as follows:

$$
\begin{aligned}
E\left[\boldsymbol{X}_{\boldsymbol{i}}\right] & =\int_{0}^{\infty} \operatorname{Pr}\left\{\boldsymbol{X}_{\boldsymbol{i}}>a\right\} d a \\
& =\int_{0}^{r}\left(1-e^{-\lambda A_{1}(i)}\right) d a \\
& =r-\int_{0}^{r} e^{-\frac{\rho A_{1}(i)}{\pi r^{2}}} d a .
\end{aligned}
$$

Note that, if the packet travels to any location within the transmission range of the destination node, only one more hop is necessary. As a result, the number of hops can be defined as $\boldsymbol{H}=\boldsymbol{H}^{\prime}+1$, where $\boldsymbol{H}^{\prime}$ is a random variable corresponding to the first $\boldsymbol{H}^{\prime}$ hops until the packet reaches a location within the transmission range of the destination node. As an approximation, we have

$$
\sum_{i=1}^{\boldsymbol{H}^{\prime}} \boldsymbol{X}_{\boldsymbol{i}} \approx v-r
$$

It should be noted that the per-hop progress $\boldsymbol{X}_{\boldsymbol{i}}, \quad i=$ $1, \ldots, \boldsymbol{H}$, at each hop are not identical and independent random variables. However, based on (21), we can observe that $E\left[\boldsymbol{X}_{\boldsymbol{i}}\right]$ never increases when $v_{i}$ decreases, because $A_{1}(i) \leq A_{1}(j)$ if $v_{i} \leq v_{j}$. Furthermore, note that the values of $E\left[\boldsymbol{X}_{\boldsymbol{i}}\right]$ at other hops are very close to $E\left[\boldsymbol{X}_{\mathbf{1}}\right]$ at the first hop due to the fact that $A_{1}(i)$ quickly converges to $A_{1}(1)$ when the distance $v_{i}$ increases. As a result, the following expression can be obtained:

$$
E\left[\boldsymbol{H}^{\prime} \mid \boldsymbol{V}=v\right] E\left[\boldsymbol{X}_{\mathbf{1}}\right] \approx E\left[\sum_{i=1}^{\boldsymbol{H}^{\prime}} \boldsymbol{X}_{\boldsymbol{i}}\right] \approx v-r
$$

where $E\left[\boldsymbol{X}_{\mathbf{1}}\right]$ is the expected per-hop progress at the first hop on an end-to-end path, as determined by (21).

Therefore, we have an approximate expression for the average number of hops, i.e.,

$$
\begin{aligned}
E[\boldsymbol{H} \mid \boldsymbol{V}=v] & =E\left[\boldsymbol{H}^{\prime} \mid \boldsymbol{V}=v\right]+1 \\
& \approx \frac{v-r}{E\left[\boldsymbol{X}_{\mathbf{1}}\right]}+1 .
\end{aligned}
$$

Substituting (18), (19), (21), and (24) into (17), we attain the following general expression for average successful delivery 


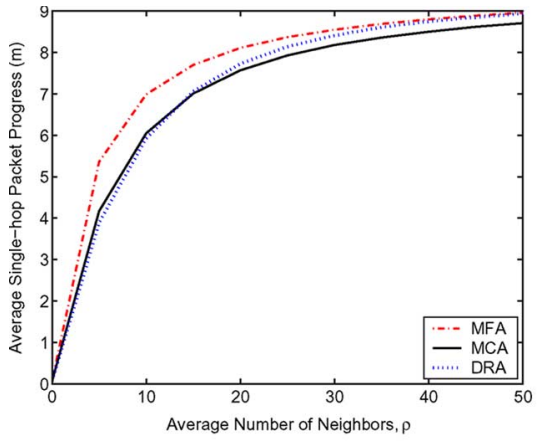

(a)

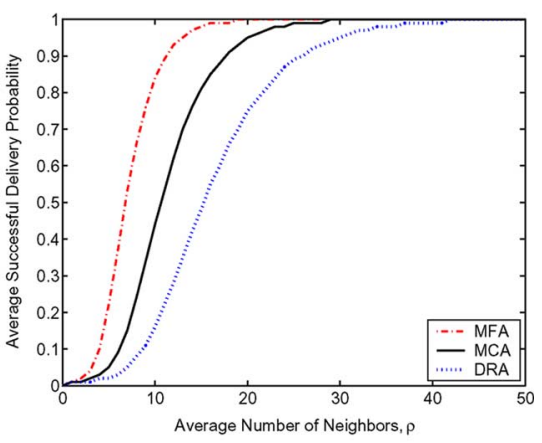

(b)

Fig. 4. Numerical results with respect to the average number of neighbors ( $r=10 \mathrm{~m}$ and $x=100 \mathrm{~m}$ ). (a) Average single-hop progress. (b) Average successful delivery probability.

probability, independent of any forwarding areas, for CGF:

$$
\begin{aligned}
& E[\boldsymbol{Z}] \approx\left(\frac{r^{2}}{x^{2}}\right) \\
& +\left(\int_{r}^{x}(1-\operatorname{Pr}\{\text { void }\})\left(\frac{v-r}{r-\int_{0}^{r} e^{-\frac{\rho A_{1}(1)}{\pi r^{2}} d a}}+1\right) \frac{2 v}{x^{2}} d v\right) .
\end{aligned}
$$

The difference between MFA, MCA, and DRA lies in the expressions of $A_{1}(1)$ (i.e., the $A_{1}$ region at the first hop), which are given in [10], and the expressions of $\operatorname{Pr}\{$ void $\}$, which are given by (5), (2) and (3), respectively. Specific expression for an individual area is given by (25) after the appropriate expressions are substituted.

\section{F. Asymptotic Behavior of Successful Delivery Probability}

When $\rho$ is reasonably large, the expected value of $\boldsymbol{Z}$ is

$$
E[\boldsymbol{Z}] \rightarrow 1, \quad \text { for large } \rho
$$

for all the three forwarding areas. Thus, average successful delivery probability is the same for all the forwarding areas and is equal to 1 . This is expected: As $\rho$ increases to a reasonably large value, it is very likely to find at least one next-hop node at each hop, so that with a probability of 1 , the packet arrives at the destination. The choice of different forwarding areas will not affect successful delivery probability.

\section{Numerical Evaluation AND Simulation Results}

In this section, we present numerical results for the performance of CGF when using MFA, MCA, and DRA. Simulation results are provided as well.

\section{A. Numerical Evaluation}

As shown in (14) and (25), the average single-hop progress and the average successful delivery probability are functions of node transmission range $r$, network range $x$, and average number of neighbors $\rho$ (or nodal density $\lambda$ ). We present our numerical results in Figs. 4-6. Fig. 4(a) compares the analytically obtained average single-hop progress, and Fig. 4(b) compares the analytically obtained average successful delivery probability for MFA, MCA, and DRA, with respect to different average numbers of neighbors. The network range is fixed to be a circle with a radius of $100 \mathrm{~m}$ (i.e., $x=100 \mathrm{~m}$ ), whereas node transmission range is set to $10 \mathrm{~m}$ (i.e., $r=10 \mathrm{~m}$ ). Note that similar results have been observed for different values of $r$ and $x$. In Figs. 5 and 6, we compare the average single-hop progress and the average successful delivery probability with respect to different network range $x$. Node transmission range is fixed at $10 \mathrm{~m}$, whereas $\rho$ is set to 5 nodes, 15 nodes, and 50 nodes, respectively.

It can be seen from Fig. 4(a) that average single-hop progress increases quickly for small $\rho$ and then slowly after $\rho$ exceeds a certain value; that is, there exists a knee in the curves, and $\rho_{\text {knee }} \approx 10-20$. The explanation of this phenomenon lies in the probability of void, as shown in Fig. 2. From Fig. 2, we can see that the probability of void decreases quickly when $\rho$ varies from 0 to $\rho_{\text {knee }}$. Such results suggest that the probability of void dominates the average single-hop progress in sparse networks (when $\rho$ is small). As a result, the curves in Fig. 4(a) are the inverse of those in Fig. 2 with a similar rate.

Another important observation from Fig. 4(a) is that, in terms of average single-hop progress, when $\rho$ is low, i.e., from 0 to around 15 nodes, MFA performs the best, then MCA, followed by DRA; when $\rho$ is medium, i.e., between 15 and 40 nodes, MFA still performs the best, followed by DRA, and they all outperform MCA; when $\rho$ is high, i.e., more than 40 nodes, DRA converges to the performance of MFA, and both are better than MCA. It is expected that the performance of MCA will converge to that of MFA as $\rho$ increases further.

The aforementioned observation can be explained as follows: When $\rho$ is small, the size of the forwarding area dominates the average single-hop progress because it determines the probability of void for that area; when $\rho$ is large enough to eliminate the possibility of voids, the location of the forwarding area dominates the calculation of average single-hop progress because a forwarding area that covers more area that is nearest to the destination will have better next-hop candidate nodes and thereby attains a larger average single-hop progress. Similar conclusions can be drawn from Fig. 5, which suggests that our observation holds for different values of network range. 


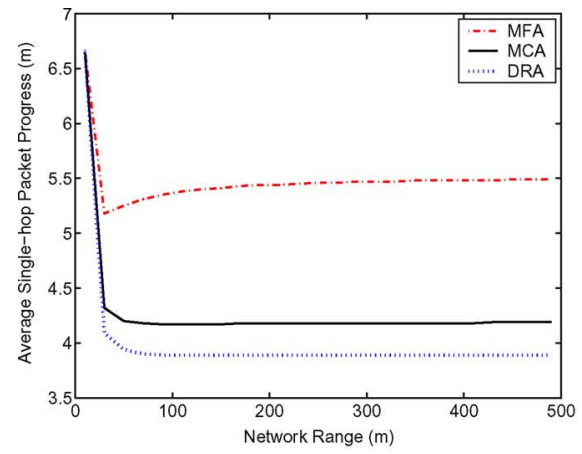

(a)

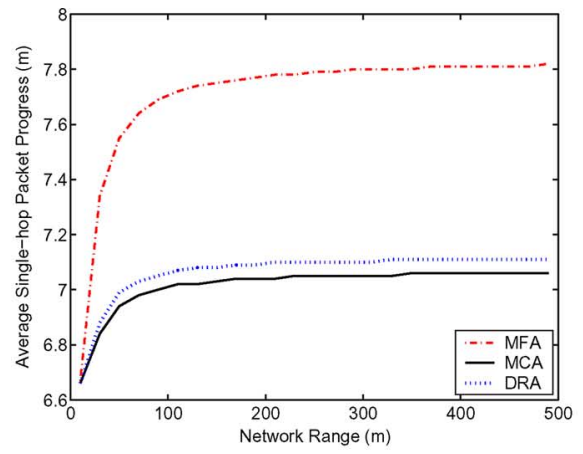

(b)

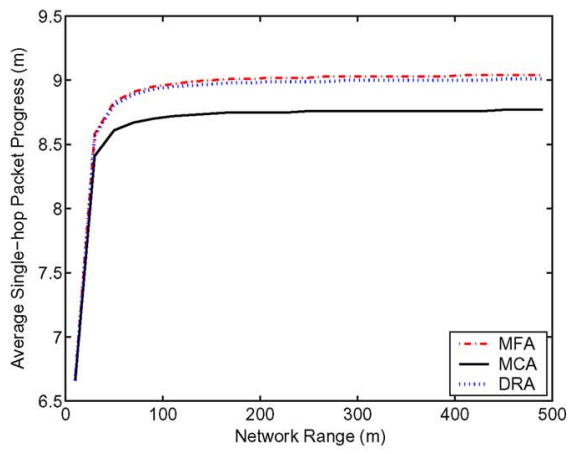

(c)

Fig. 5. Numerical results for average single-hop progress versus network range. (a) $r=10 \mathrm{~m}$ and $\rho=5$ nodes. (b) $r=10 \mathrm{~m}$ and $\rho=15$ nodes. (c) $r=10 \mathrm{~m}$ and $\rho=50$ nodes.

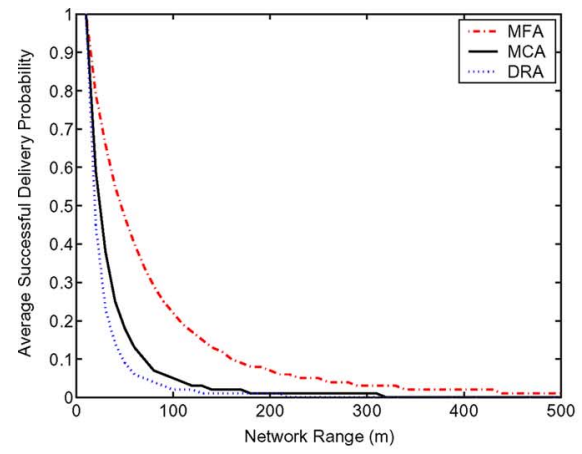

(a)

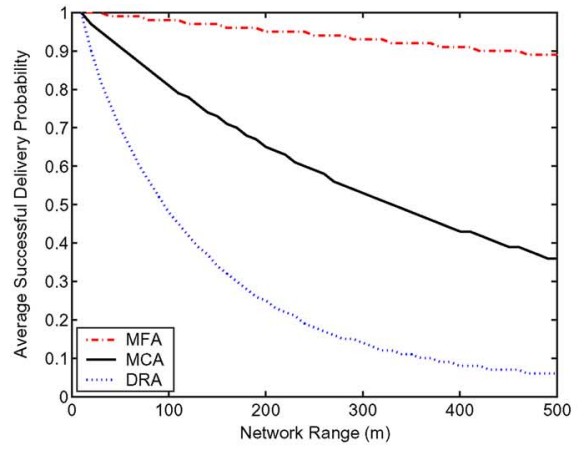

(b)

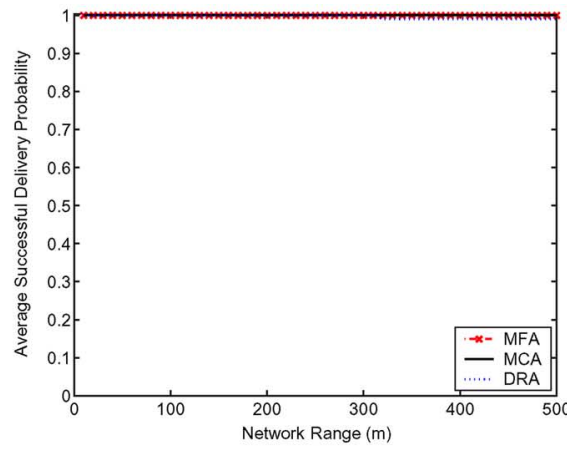

(c)

Fig. 6. Numerical results for average successful delivery probability versus network range. (a) $r=10 \mathrm{~m}$ and $\rho=5$ nodes. (b) $r=10 \mathrm{~m}$ and $\rho=15$ nodes. (c) $r=10 \mathrm{~m}$ and $\rho=50$ nodes.

From Fig. 5, we observe that, when $\rho=5$ and $\rho=15$, MFA attains an average single-hop progress almost $1 \mathrm{~m}$ more than MCA and DRA (a $10 \%$ performance gain). When $\rho=50$, MFA and DRA achieve a $0.5 \mathrm{~m}$, i.e., $5 \%$, more average single-hop progress than MCA. In the case of $\rho=5$, when network range is small and is comparable to node transmission range, average single-hop progress drops quickly and then maintains a lower value regardless of $x$. In the case of $\rho=15$ or $\rho=50$, this trend is reversed, and average single-hop progress jumps to a higher level and stays at that level as $x$ increases. This result can be explained with our three-step forwarding strategy: When network range is small, only step 1 affects the result; however, when network range is much larger than node transmission range, steps 2 and 3 also impact the result. When $\rho=5$, step 3 has a larger impact than step 2, so the initial value is larger than the stable value. In the case of $\rho=15$ or $\rho=50$, step 2 instead has a larger impact than step 3 , and the initial value is thereby lower than the stable value.

In terms of the average successful delivery probability, it can be seen from Fig. 4(b) that it climbs up very quickly for small $\rho$ and then slowly after $\rho$ exceeds a certain value (i.e., a knee exists in the curves), which is similar to the average singlehop progress. Unlike the average single-hop progress, $\rho_{\text {knee }}$ of the average successful delivery probability is different for the three forwarding areas. For MFA, $\rho_{\text {knee }} \approx 10-20$, whereas $\rho_{\mathrm{knee}} \approx 15-25$ for MCA, and $\rho_{\mathrm{knee}} \approx 25-35$ for DRA. The explanation of this phenomenon is related to the probability of void and the average number of hops, as shown in (25).
Fig. 2 shows that there exists a knee $\left(\rho_{\text {knee }} \approx 10-20\right)$ for all three forwarding areas in terms of the probability of void. The location of the knee is further magnified by the average number of hops. Since MFA has the lowest probability of void due to the largest forwarding area, this effect is not very significant as compared to MCA and DRA. Such results illustrate how the void problem affects the packet delivery over multiple hops. Furthermore, we note that in terms of the average successful delivery probability, MFA always performs the best, then MCA, followed by DRA. As expected, the performance of MCA and DRA converges to that of MFA (i.e., a packet will arrive at the destination node with a probability of 1) as $\rho$ increases beyond a certain value. For MCA, the convergence value of $\rho$ is around 30, whereas the value is around 40 for DRA, where their probabilities of void are almost 0 .

As shown in Fig. 6, we observe that, when $\rho=5$ and $\rho=15$, all the curves fall down when the network range increases. The main reason is that when the network range increases, the average number of hops needed increases due to a larger average distance between the source node and the destination node, which leads to a higher possible packet loss and, thus, to a lower average successful delivery probability. When $\rho=50$, the curves are straight lines close to 1 , and they look almost identical because the probability of void is close to 0 , and thus, there is no packet loss any more. Note that when $\rho$ is low, i.e., $\rho=5$, the average successful delivery probability is very low as well, there only exists a slight difference among MFA, MCA, and DRA, whereas MFA performs the best. When $\rho$ is 


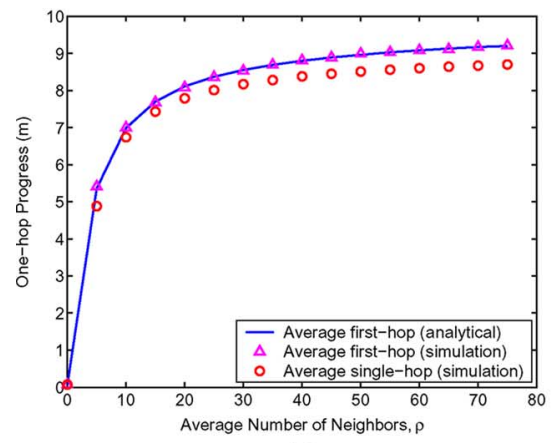

(a)

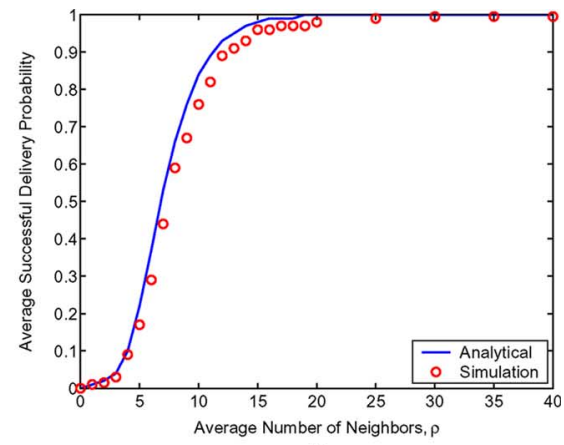

(b)

Fig. 7. Simulations results for the validation of our analysis ( $r=10 \mathrm{~m}, x=100 \mathrm{~m}$, MFA). (a) Average single-hop progress. (b) Average successful delivery probability.

medium, i.e., $\rho=15$, the curves show that MFA has a much better performance than MCA and DRA, which shows that MFA, under such network density, is much more robust than MCA and DRA for CGF in the presence of voids.

All the aforementioned observations provide guidance for the selection of a forwarding area for the design of a practical CGF protocol in a randomly distributed wireless multi-hop network. ${ }^{3}$ That is, in terms of the two performance metrics and under the assumptions considered in this paper, if $\rho$ is known a priori to be low or medium, we should select MFA as the forwarding area; if $\rho$ is larger than 40, DRA should be selected because it enables a simpler design and operation of a distributed contention scheme as well as a lighter protocol processing burden at nodes [4]-[6] and, at the same time, providing similar network performance. MCA should be used only when $\rho$ is extremely high (e.g., $\rho>100$ ).

\section{B. Simulation Results}

Simulations (programs written in $\mathrm{C}$ language and run in Microsoft Visual C++ 6.0) have been performed to validate our analytical framework. In our simulations, the network nodes are distributed in a circular region according to a 2-D Poisson distribution. The circle is centered at $(0,0)$ with a radius of $100 \mathrm{~m}$, whereas node transmission range is set to $10 \mathrm{~m}$. The source node is fixed at $(0,0)$, whereas destination nodes are randomly chosen in the circle. The source node transmits packets to the selected destination node in accordance to our three-step forwarding strategy while specifying a forwarding area. We measured the average first-hop progress, the average single-hop progress (not only the first hop but also the average of all the hops from the source node to the destination node), and the average successful delivery probability, as shown in Fig. 7. All the results are the average of 500 runs, each of which selects 100 destinations randomly.

Fig. 7(a) compares the average first-hop progress and the average single-hop progress obtained by simulations with numerical results calculated from (14) for MFA. Results for MCA and DRA are similar and are not shown here. While simulation results on average first-hop progress match our numerical

\footnotetext{
${ }^{3}$ Note that the conclusion may be invalid if some assumptions are violated, which are further discussed in Section V.
}

results very well, the overall average single-hop progress is slightly lower than our analytically derived average first-hop progress. The main reason is that the last-hop progress, where a packet is directly delivered to a destination, is often less than the progress in the previous hops, where a packet is greedily forwarded. We have executed extensive simulations on the overall average single-hop progress for different forwarding areas. Conclusions similar to those in Fig. 4(a) can be drawn regarding the performance of MFA, MCA, and DRA. In fact, the effect of last-hop progress is similar for different forwarding areas. Thus, we can use the analytically derived average firsthop progress as the average single-hop progress to evaluate CGF with different forwarding areas.

In Fig. 7(b), we compare the average successful delivery probability obtained by simulations with numerical results calculated from (25) for MFA. Similar results have been obtained in the case of MCA and DRA. From the graph, we observe that simulation results are slightly lower than our analytically derived results, which means that our estimation is a little conservative. This is expected: In (24), $v-r$ is slightly smaller than the actual overall progress on an end-to-end path, whereas $E\left[\boldsymbol{X}_{\mathbf{1}}\right]$ is a little bit larger than $E\left[\boldsymbol{X}_{\boldsymbol{i}}\right]$ at other hops. In other words, the average number of hops we used is a lower bound for the actual values. As a result, our estimate of the average successful delivery probability in (25) is an upper bound. It can be seen from the graph that the bound is rather tight. Our extensive simulation results further verify that our estimates are very close to the actual results for all three forwarding areas, and thus, the upper bound is actually a tight bound. Accordingly, we can use (25) to estimate the actual average successful delivery probability and to evaluate the reliability performance of packet delivery of CGF with different forwarding areas.

\section{Discussion}

The analytical framework we presented in Section III is based on several important assumptions. In this section, we investigate the impact of these assumptions on our theoretically derived results.

\section{A. Void Handling Schemes}

Our previous study assumes that no void handling scheme is in use, and our results show that the void problem seriously 


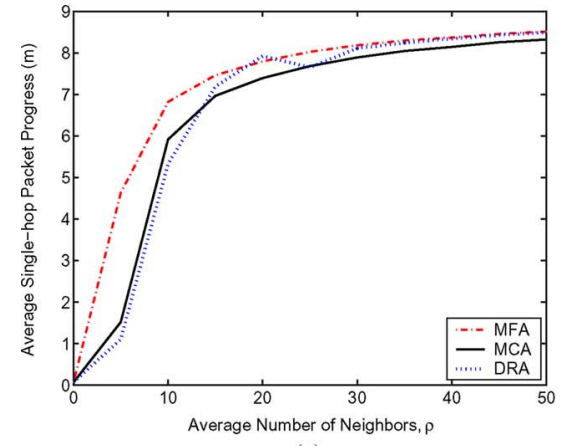

(a)

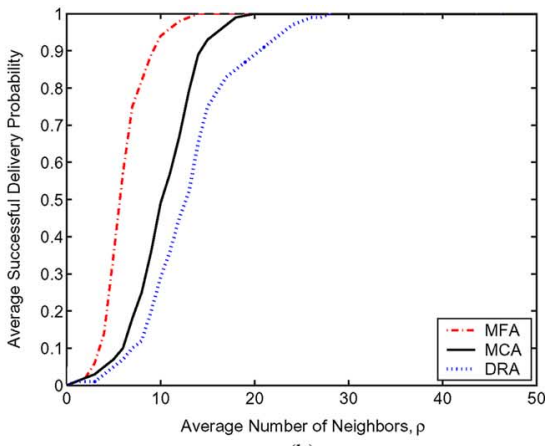

(b)

Fig. 8. Simulation results for CGF plus greedy void handling ( $r=10 \mathrm{~m}$ and $x=100 \mathrm{~m}$ ). (a) Average single-hop progress. (b) Average successful delivery probability.

affects the performance of average single-hop progress and average successful delivery probability when $\rho$ is low or medium, which necessitates the use of a void handling scheme in sparse networks. When $\rho$ is high, e.g., higher than 40, the probability of void drops to almost 0 , and the performance of CGF will not be affected by voids. In this section, we investigate how the performance of CGF varies when a void handling scheme is employed.

Note that voids can be handled by state-based void handling schemes such as perimeter routing in GPSR [9], BOUNDHOLE [8], and cost-based forwarding as in PAGER-M [16], but these techniques require nodes to collect local topology information and are state based. In this paper, we study some schemes that are state free and on demand in nature, which are a better fit for CGF, because CGF as a whole is designed as a state-free communication paradigm that leads to many benefits such as robustness to high network dynamics. ${ }^{4}$ In particular, we focus on two schemes: passive participation [4], [6] and void avoidance in SPEED [15], although other schemes such as onehop flooding [14] exist. A survey of the existing void handling schemes in detail is available in [17].

Passive participation and void avoidance belong to the same void handling approach, and if they are both executed without any failure, they have the same effect on handling voids for CGF. In this paper, we call this approach greedy void handling. The main idea of greedy void handling is that once a node cannot locate a next-hop node, it either simply discards the data packet and keeps itself from volunteering to forward any subsequent data packets toward the destination (in passive participation) or actively sends a packet to its upstream node to signal the existence of a void so that the following packets are forwarded to other nodes instead of the void node (in void avoidance). Such an approach has a reverse-propagation effect, which eventually informs other intermediate nodes to avoid those nodes with voids for the destination. It was argued in [15] that the approach is guaranteed to find a greedy path if one exists, although it is not guaranteed to find a topologically valid path. Thus, unlike GPSR [9], this approach, in theory, cannot guarantee packet delivery in a connected network.

\footnotetext{
${ }^{4}$ State free here means that a node does not store the information or states of other nodes to handle void. The on-demand feature means that such handling only takes place on a node with void upon receipt of a data packet.
}

The impact of greedy void handling on the CGF performance is shown in Fig. 8. From the figure, we can see that CGF that uses greedy void handling is rather ineffective, and it performs even worse than CGF without void handling under very low $\rho$ because this void handling approach can cause more serious network partition. However, when $\rho$ is moderate (i.e., larger than 10), it begins to function better and better and improves both performance metrics. As a result, under a network with a moderate node density, this approach can be used to handle voids for CGF due to its extra advantages of being simple, efficient, state free, and easy to implement. Furthermore, Fig. 8 also shows similar observations, as presented in Section IV-A. However, the threshold for the selection of a forwarding area mentioned in Section IV-A changes from 40 to 30. We emphasize that the conclusion about the selection of the forwarding area will change if a different void handling scheme is used.

\section{B. Next-Hop Node Selection Criterion}

Criteria similar to those used in traditional geographic forwarding with a centralized selection of the next-hop node at the sender may also be applied to CGF with a distributed selection of the next-hop node among next-hop candidate nodes. These criteria [1] include most forward within $r,{ }^{5}$ nearest with forward progress, compass routing, and random selection. Some nongeographic criteria such as energy [5]-[7] and link reliability can also be exploited for different optimization purposes.

In this paper, we simulated the performance of random selection as an illustrative example, where one of the nodes in the forwarding area is randomly selected to serve as the nexthop node. The results are shown in Fig. 9. In Fig. 9(a), when $\rho$ is larger than 10, DRA performs the best, then MCA, followed by MFA, in terms of average single-hop progress. In fact, when the random selection technique is used, the average single-hop progress can be calculated as the average of progresses when each of the nodes in the forwarding area serves to forward the packet. This is different from the model on which (14) is based. It is rather surprising to see, in Fig. 9(b), that DRA has the worst performance in terms of average successful delivery probability among the three, because DRA requires the least number of

\footnotetext{
${ }^{5}$ This is the same as distance-based greedy criterion
} 


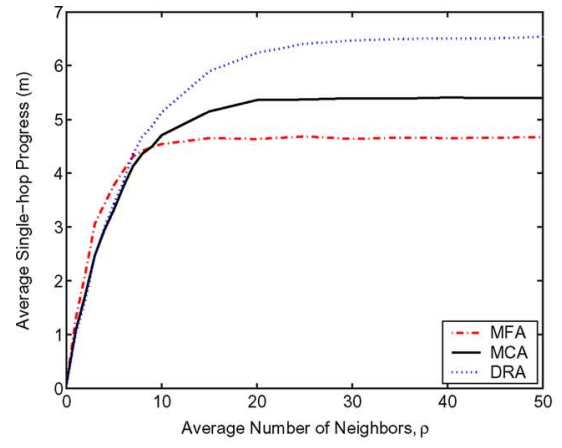

(a)

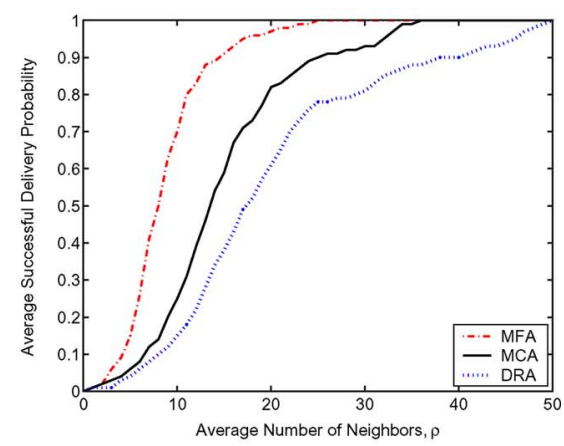

(b)

Fig. 9. Simulation results for CGF with random selection of a next-hop node within a forwarding area $(r=10 \mathrm{~m}$ and $x=100 \mathrm{~m})$. (a) Average single-hop progress. (b) Average successful delivery probability.

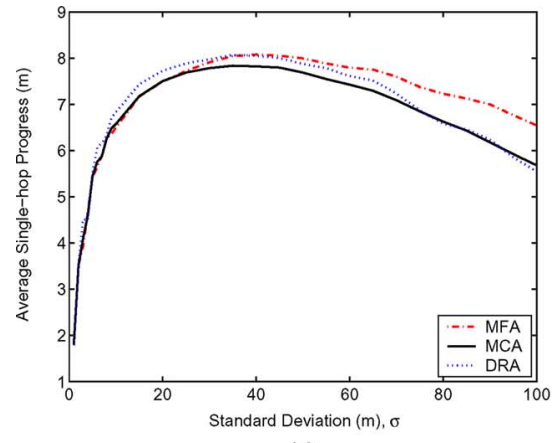

(a)

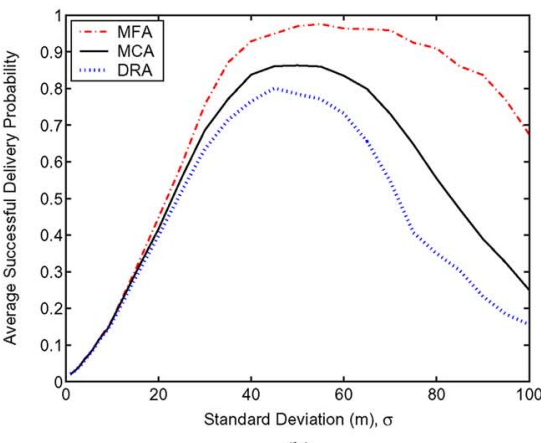

(b)

Fig. 10. Simulation results for CGF under 2-D Gaussian node distribution ( $r=10 \mathrm{~m}$ and 2000 nodes). (a) Average single-hop progress. (b) Average successful delivery probability.

hops to the destination node when $\rho$ is larger than 10. The reason is that, when $\rho$ reaches 10 , the probability of void in MFA becomes close to 0, whereas in MCA or DRA, it still has a certain value that cannot be ignored. As a result, the average number of hops have a much more significant impact on the average successful delivery probability of DRA and MCA than that of MFA. Also note that the average successful delivery probability for all the three forwarding areas in Fig. 9(b) reaches 1 at a larger $\rho$ value as compared with Fig. 4(b), because the average number of hops between the source node and the destination node increases using the random selection strategy.

The conclusion about the selection of the forwarding area may be drawn as follows: When $\rho$ is low (i.e., below 10), MFA should be chosen due to the benefit of average successful delivery probability; when $\rho$ is between 10 and 50, the selection depends on the optimization criterion for the network. On one hand, MFA has the best average successful delivery probability, whereas on the other hand, DRA has the best average singlehop progress, which may lead to the best average network delay performance. When $\rho$ is larger than 50, DRA is preferred due to the benefit of average single-hop progress.

\section{2-D Gaussian Node Distribution}

To evaluate the CGF performance under a node distribution with nonuniform node density, we assume that nodes follow a 2-D Gaussian distribution, which is centered at $(0,0)$; that is, the mean of the Gaussian distribution equals $(0,0)$. The probability density function (pdf) for the location of any node is determined by [13]

$$
f(x, y)=\frac{1}{2 \pi \sigma^{2}} e^{-\left(x^{2}+y^{2}\right) / 2 \sigma^{2}}
$$

where $-\infty \leq x, y \leq \infty$, and $\sigma$ is the standard deviation.

In our simulation, 2000 nodes, which follow the aforementioned distribution, are deployed on a very large field, ${ }^{6}$ whereas we vary the value of the standard deviation in different runs. The source node is fixed at $(0,0)$, whereas destination nodes are randomly chosen within the circle of a radius of $100 \mathrm{~m}$. Obviously, the node density in a unit area in this distribution is not expected to be a constant any more. The region nearer to the origin, where the source resides, has a higher node density. The simulation results are presented in Fig. 10.

From the graphs, we can see that all of the three forwarding areas show the same tendency. That is, when $\sigma$ is small, the performance goes up when $\sigma$ increases; the curve attains a maximum value at a medium value of $\sigma$; and later, it gradually decreases when $\sigma$ continues to increase. However, the medium value of $\sigma$ is different: It is $30-40$ for average single-hop progress in Fig. 10(a) and 40-60 for average successful delivery probability in Fig. 10(b). This can be explained as follows: When $\sigma$ is small, most nodes reside in a small circular area very near to the center (sometimes only within one hop, i.e., within a radius of $10 \mathrm{~m}$ ) and few nodes span farther. When destination

\footnotetext{
${ }^{6}$ The field is considered to be very large so that the Gaussian pdf can be employed here to approximate node deployment.
} 


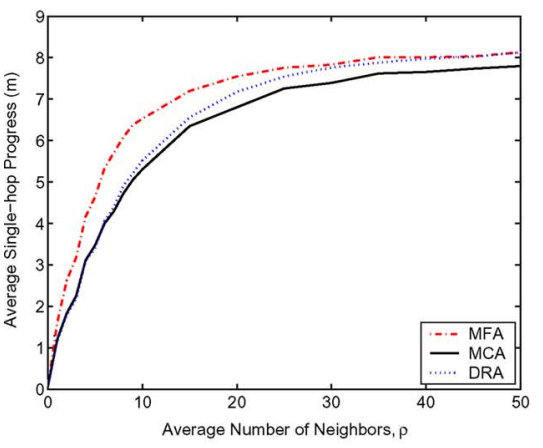

(a)

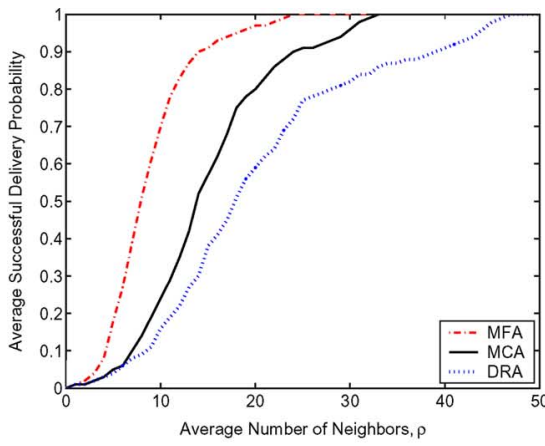

(b)

Fig. 11. Simulation results for CGF under Gaussian location error (zero mean, standard deviation $\sigma=0.5, r=10 \mathrm{~m}$, and $x=100 \mathrm{~m}$ ). (a) Average single-hop progress. (b) Average successful delivery probability.

node is within or very close to this very high density area, data packets can be delivered. However, when the destination node is chosen out of this area, most data packets will be dropped after the first few hops. When $\sigma$ increases, more nodes begin to span into the region where the destination node can reside $(x=100 \mathrm{~m})$. Thus, the performance values also rise. When $\sigma$ further increases, more and more nodes can be deployed outside the circular area of radius $x$; that is, the node density within the network range $x$ area gradually decreases on average, which leads to the decreasing performance values.

In general, MFA shows the best performance. The result suggests that as long as we still employ the node selection using distance-based greedy criterion, MFA always performs the best regardless of how nodes are deployed. However, the degree of performance gap among different forwarding areas is related to the specific node distribution model. Note that a 2-D Gaussian node distribution is not homogeneous any more. Thus, average first-hop progress cannot be employed to approximate to average single-hop progress. In fact, it is expected that the average first-hop progress will be much higher than the average single-hop progress because the area within the first hop always has a higher node density than other areas. Meanwhile, our analytical results on the average successful delivery probability are not applicable as well.

\section{Location Errors}

Our study assumes that all nodes in the network can obtain the location information without errors. However, in practice, high-precision location information is very difficult or highly expensive to achieve. In addition, location errors are unavoidable even in the most current advanced localization techniques. This section examines the impact of location errors on the performance of CGF for three forwarding areas. We have executed the simulation for a scenario in which the selection of a next-hop node is done based on a perturbed version of the actual nodes' locations. More specifically, we introduced Gaussian errors into the actual coordinates of the nodes, with zero mean and standard deviation $\sigma=0.5$.

The results of Fig. 11 show that the performance degradation introduced by location errors is modest even when $\sigma=0.5$. It can be seen that average single-hop progress in Fig. 11(a) is about 5\% to $15 \%$ less than that in Fig. 4(a), which implies that a modestly larger number of average number of hops between the source node and the destination node is required, which leads to a performance degradation of average successful delivery probability shown in Fig. 11(b) as compared to that in Fig. 4(b). Also note that in Fig. 11(a), DRA has a better performance in terms of average single-hop progress than MCA at a smaller $\rho$ than the performance curves in Fig. 4(a). The main reason comes from the next-hop node selection using nodes' locations with errors, because it is now likely for a node to be actually farther away from the destination node, instead of a node that is actually closest to the destination node, to be a next-hop node. Location errors may also lead to a larger probability of void and, possibly, negative progress at some hops.

Furthermore, since the location errors we simulated have a similar impact on the performance of the three forwarding areas, our conclusion about the selection of a forwarding area in Section IV-A remains valid. However, the node density threshold for the selection of a specific forwarding area is changed from 40 to 45 in this scenario. The conclusion will change if a different $\sigma$ value or a different location error model is used.

\section{E. Contention Resolution Schemes}

Our study has assumed a perfect contention scheme that can effectively establish a single next-hop node in a forwarding area. Such a contention scheme in CGF can be designed more easily, and the protocol processing can generate a lighter load at nodes when MCA or DRA is used than when MFA is used, because all the contending nodes in MCA or DRA are within the communication range of each other [4]-[6].

When the contention scheme in use cannot resolve the contentions successfully, either none or more than one next-hop nodes will be established. Such a situation becomes rather complicated for tractable analysis and is considered beyond the scope of this paper. Intuitively, when more than one next-hop nodes are established, the reliability of packet delivery of CGF will improve at the cost of more resources being used.

\section{CONCLUSION}

In this paper, we have constructed a general analytical framework, in terms of average single-hop progress and average 
successful delivery probability, to investigate several research issues related to a predefined forwarding area in CGF. Our results provide guidelines for the selection of a forwarding area for the design of a CGF protocol. The analytical framework, which is validated by numerical results and extensive simulations, also serves as a performance evaluation technique for a broad range of geographic forwarding protocols.

Note that we have not explicitly analyzed the energy consumption of CGF in the framework. Indeed, CGF has the same energy consumption rate at senders for all three forwarding areas because the transmission power always remains the same due to the fixed transmission range in CGF, and hence, the average single-hop progress directly affects the energy consumption in the sense of the average number of transmissions (or hops) required in the network. We believe that the performance comparison of the energy consumption of CGF for the three forwarding areas is implicit when we considered the average single-hop progress. An in-depth analysis of energy consumption is only possible when the MAC scheme in use is clearly specified, which would be our future work. It would also be interesting to perform some packet-level simulations on the existing CGF protocols to compare the results with those obtained from our framework.

\section{REFERENCES}

[1] M. Mauve, J. Widmer, and H. Hartenstein, "A survey on position-based routing in mobile ad hoc networks," IEEE Netw., vol. 15, no. 6, pp. 30-39, Nov. 2001

[2] M. Heissenbüttel, T. Braun, T. Bernoulli, and M. Wälchli, "BLR: Beaconless routing algorithm for mobile ad-hoc networks," Comput. Commun. J., vol. 27, no. 11, pp. 1076-1086, Jul. 2004.

[3] H. Füssler, J. Widmer, M. Käsemann, M. Mauve, and H. Hartenstein, "Contention-based forwarding for mobile ad-hoc networks," Ad Hoc Netw. J., vol. 1, no. 4, pp. 351-369, Nov. 2003.

[4] M. Zorzi and R. R. Rao, "Geographic random forwarding (GeRaF) for ad hoc and sensor networks: Multihop performance," IEEE Trans. Mobile Comput., vol. 2, no. 4, pp. 337-348, Oct.-Dec. 2003.

[5] B. M. Blum, T. He, S. Son, and J. A. Stankovic, "IGF: A robust state-free communication protocol for sensor networks," CS Dept., Univ. Virginia, Charlottesville, Tech. Rep. CS-2003-11, 2003.

[6] D. Chen, J. Deng, and P. K. Varshney, "A state-free data delivery protocol for wireless sensor networks," in Proc. IEEE WCNC, New Orleans, LA, Mar. 2005, pp. 1818-1823.

[7] D. Ferrara, L. Galluccio, A. Leonardi, G. Morabito, and S. Palazzo, "MACRO: An integrated MAC/Routing protocol for geographic forwarding in wireless sensor networks," in Proc. IEEE Infocom, Miami, FL, Mar. 2005, pp. 1770-1781.

[8] Q. Fang, J. Gao, and L. J. Guibas, "Locating and bypassing routing holes in sensor networks," in Proc. IEEE Infocom, Hong Kong, Mar. 2004, pp. 2458-2468.

[9] B. Karp and H. T. Kung, "Greedy perimeter stateless routing for wireless networks," in Proc. ACM MobiCom, Boston, MA, Aug. 2000, pp. 243-254.

[10] D. Chen, J. Deng, and P. K. Varshney, "On the forwarding area of contention-based geographic forwarding for ad hoc and sensor networks," in Proc. IEEE SECON, Santa Clara, CA, Sep. 2005, pp. 130-141.

[11] J. Deng, Y. S. Han, P.-N. Chen, and P. K. Varshney, "Optimum transmission range for wireless ad hoc networks," in Proc. IEEE WCNC, Atlanta, GA, Mar. 2004, pp. 1024-1029.

[12] P. Billingsley, Probability and Measure. New York: Wiley, 1995.

[13] A. Leon-Garcia, Probability and Random Processes for Electrical Engineering, 2nd ed. Reading, MA: Addison-Wesley, 1994.

[14] I. Stojmenovic and X. Lin, "Loop-free hybrid single-path/flooding routing algorithms with guaranteed delivery for wireless networks," IEEE Trans. Parallel Distrib. Syst., vol. 12, no. 10, pp. 1023-1032, Oct. 2001.

[15] T. He, J. A. Stankovic, C. Lu, and T. Abdelzaher, "SPEED: A stateless protocol for real-time communication in sensor networks," in Proc. Int. Conf. Distrib. Comput. Syst., Providence, RI, May 2003, pp. 46-55.
[16] L. Zou, M. Lu, and Z. Xiong, "PAGER-M: A novel location-based routing protocol for mobile sensor networks," in Proc. Broadwise. San Jose, CA, Oct. 2004.

[17] D. Chen and P. K. Varshney, "A survey of void handling techniques for geographic routing in wireless networks," Commun. Surveys Tuts., vol. 9, no. 1, pp. 50-67, First Quarter 2007.

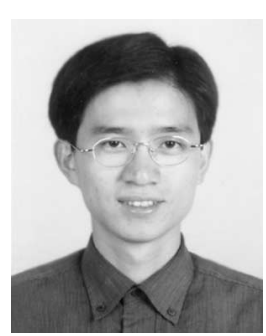

Dazhi Chen (S'05-M'06) received the B.E. and M.E. degrees from Nanjing University of Posts and Telecommunications, Nanjing, China, in 1998 and 2001, respectively, and the Ph.D. degree in electrical engineering from Syracuse University, Syracuse, NY, in 2007.

$\mathrm{He}$ is currently a Research Associate with the Department of Electrical Engineering and Computer Science at Syracuse University, where he was a Graduate Fellow during 2001-2004. His research interests are in wireless communications networking, mobile computing, and network security. His current research focuses on the design and evaluation of communications, networking, and security techniques for next-generation wireless networks.

Dr. Chen is a member of the Association for Computing Machinery.

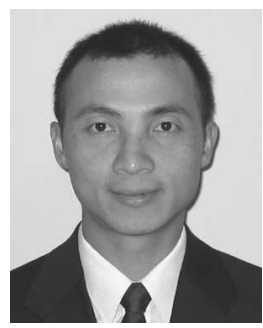

Jing Deng (S'98-M'02) received the B.E. and M.E. degrees in electronic engineering from Tsinghua University, Beijing, China, in 1994 and 1997, respectively, and the Ph.D. degree in electrical and computer engineering from Cornell University, Ithaca, NY, in 2002.

$\mathrm{He}$ is an Assistant Professor with the Department of Computer Science, University of New Orleans, New Orleans, LA. From 2002 to 2004, he visited the Computer Applications and Software Engineering Center and the Department of Electrical Engineering and Computer Science, Syracuse University, Syracuse, NY, as a Research Assistant Professor, supported by the Syracuse University Prototypical Research in Information Assurance program. He was a Teaching Assistant from 1998 to 1999 and a Research Assistant from 1999 to 2002 with the School of Electrical and Computer Engineering, Cornell University. His research interests include mobile ad hoc networks, wireless sensor networks, wireless network security, energy-efficient wireless networks, and information assurance.

Dr. Deng is a member of the Association for Computing Machinery. He has served as the Co-Chair of the IEEE International Workshop on Ad Hoc and Ubiquitous Computing (AHUC'06), Taiwan, R.O.C., and the Sponsorship Chair of the First International Conference on Multimedia Services Access Networks (MSAN'05), Orlando, FL. He has served on the Technical Program Committees of many IEEE conferences, including MASS'05, MASS'06, GLOBECOM'06, and WCNC'07.

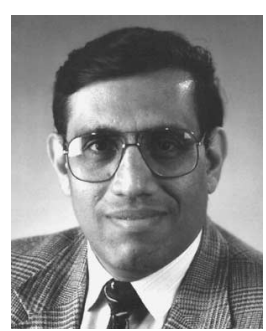

Pramod K. Varshney (S'72-M'77-SM'82-F'97) received the B.S. degree in electrical engineering and computer science (with highest honors) and the M.S. and $\mathrm{Ph} . \mathrm{D}$. degrees in electrical engineering from the University of Illinois at Urbana-Champaign in 1972, 1974, and 1976, respectively.

Since 1976, he has been with Syracuse University, Syracuse, NY, where he is currently a Professor of electrical engineering and computer science and the Research Director of the New York State Center for Advanced Technology in Computer Applications and Software Engineering. His current research interests are in distributed sensor networks and data fusion, detection and estimation theory, wireless communications, image processing, radar signal processing, and remote sensing.

Prof. Varshney serves as a Distinguished Lecturer for the IEEE Aerospace and Electronic Systems Society. In 2001, he was the President of the International Society of Information Fusion. He was a recipient of numerous awards. 